\title{
Modeling for the Calcination Process of Industry Rotary Kiln Using ANFIS Coupled with a Novel Hybrid Clustering Algorithm
}

\author{
Yongchang Cai ${ }^{1,2}$ \\ ${ }^{1}$ School of Electronics and Information Engineering, Shunde Polytechnic, Foshan 528300, China \\ ${ }^{2}$ Technical University of Dresden, 01062 Dresden, Germany \\ Correspondence should be addressed to Yongchang Cai; herocych@163.com
}

Received 4 December 2016; Accepted 12 February 2017; Published 2 March 2017

Academic Editor: Tarek Ahmed-Ali

Copyright ( $\odot 2017$ Yongchang Cai. This is an open access article distributed under the Creative Commons Attribution License, which permits unrestricted use, distribution, and reproduction in any medium, provided the original work is properly cited.

Rotary kiln is important equipment in heavy industries and its calcination process is the key impact to the product quality. Due to the difficulty in obtaining the accurate algebraic model of the calcination process, an intelligent modeling method based on ANFIS and clustering algorithms is studied. In the model, ANFIS is employed as the core structure, and aiming to improve both its performance in reduced computation and accuracy, a novel hybrid clustering algorithm is proposed by combining FCM and Subtractive methods. A quasi-random data set is then hired to test the new hybrid clustering algorithm and results indicate its superiority to FCM and Subtractive methods. Further, a set of data from the successful control activity of sophisticated workers in manufacturing field is used to train the model, and the model demonstrates its advantages in both fast convergence and more accuracy approaching.

\section{Introduction}

Calcination process is omnipresent in heavy industries worldwide, such as chemical industry, steel manufactory, and metallurgical industry. This process is significantly important for the final product quality because the calcination is where the product changed its form physically or chemically under certain temperature for a certain span of time. Featuring nonlinearity, long time delay, multivariables and their serious coupling, and a lot of control theories and modeling methodologies for rotary kiln have been studied in the past few decades [1-3].

Some researchers built up the algebraic models of rotary kiln by analyzing the gas flow, granular material flow, and heat transfer. The approaches they used were mainly based upon aerodynamics and mechanical structure [4-7]. However, for a specific production kiln, it is usually difficult to obtain the necessary parameters for the adequately accurate model, which is a bottleneck for them to be generalized in wider applications.
In the past decade, many researches have been carried out on the rotary kiln control based on intelligent and prediction techniques. For instance, expert system was proposed to control the kiln which improved the production outcome $[7,8]$. Soft modeling methods based on neural network, support vector machines, and subspace method were used to predict the output index, the calcination temperature, and tail temperature of the kiln, respectively [9-11]. However, there are still many problems among those studies such as bulky computation and excessive restrictions, and also researches on the modeling for the calcination process of the kiln, which is the core factor for the product quality, are rarely reported.

ANFIS (Adaptive Network-based Fuzzy Inference System) as a model identification method has drawn much attention in different application fields recently [12-14]. Compared with conventional techniques, it has the advantages of mapping all the inputs to the corresponding outputs based only on the available data and incorporating linguistic knowledge for problem solving and strong generalization capability. 


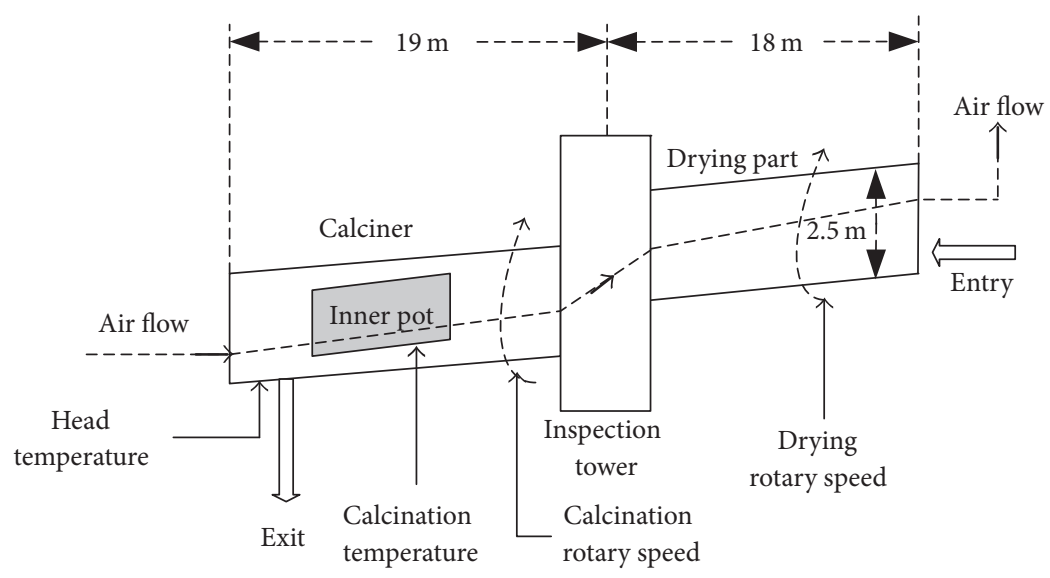

FIGURE 1: Schematic diagram of the rotary kiln.

In order to improve the computation efficiency and identifying ability of ANFIS, clustering algorithm is utilized to partition the data into clusters and generate appropriate number of fuzzy rules. Among many clusters, fuzzy $C$-means Clustering Method (FCM) [15] and Subtractive Clustering Method (Subclust) [16] are widely adopted. But each of them has its drawback. Subclust only yields the approximations for the actual cluster centers, whereas for FCM, the number of clusters has to be decided empirically and the algorithm is sensitive to randomly initiated membership grade. It means there is no enough guarantee to find the actual centers for the clusters by applying each of the two clustering methods. Overcoming these problems is significant because tiny deviation of the clustering centers leads to apparent difference in the identified model when the training data have high dimension and they are not so explicitly distinguished.

In this paper, ANFIS is employed as the core structure for the calcination control model with the input and output variables selected by analyzing the calcination reaction and the experience of sophisticated workers. As a premise procedure to modeling, a hybrid clustering algorithm combing FCM and Subclust is put forwards which gets over the weaknesses of FCM and Subclust and leads to more accuracy of the cluster centers.

The rest of this paper starts with an introduction to the industrial rotary kiln and its calcination process in Section 2. In Section 3, FCM and Subclust methods are introduced and then a novel hybrid clustering way of combining these two clustering algorithms is proposed and illustrated in detail. Section 4 presents ANFIS concisely which is adopted as a core modeling structure for the calcination process of the kiln in the next section. In Section 5, modeling is conducted with the method of ANFIS coupled with the new hybrid clustering algorithm, and the implementation results are discussed.

\section{The Rotary Kiln and Calcination Process}

The rotary kiln to be studied in this paper is composed of two cylinders, calciner, and drying part, which are connected by an inspection tower. It is actually gigantic equipment with

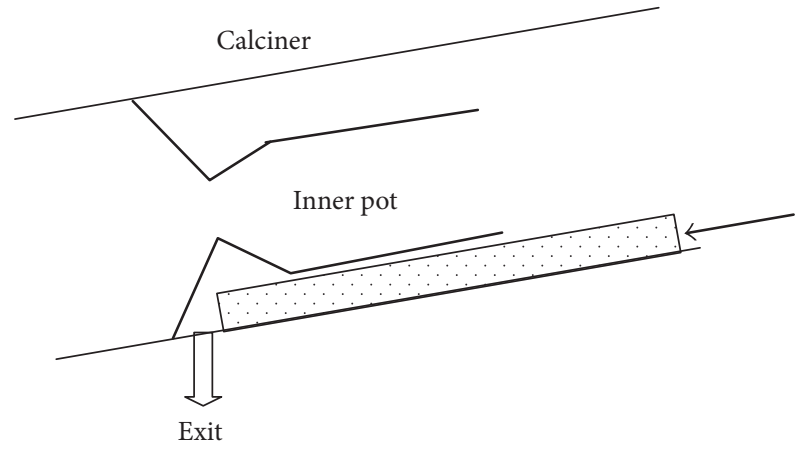

FIGURE 2: Schematic diagram of the inner pot.

length of 37 meters and a diameter of 2.5 meters, as seen in Figure 1. The kiln is installed with a slope of around $5^{\circ}$ and it rotates around its axis. The drying part has similar length as the calciner, acting as a preheater for the inner material [17].

The material going through the kiln is lithopone, an inorganic compound, used as a white pigment. It is first fed into the elevated cold end, the right side of the drying part, and as the kiln rotates it moves along the declining inner bed due to gravity, towards the exit which is at the left side of calciner. During the long inner rolling, the material is first preheated in drying part, where the temperature is $150^{\circ} \mathrm{C} \sim$ $200^{\circ} \mathrm{C}$, and then goes into the calciner which includes an inner pot, as shown in Figure 2. The temperature around the pot is relatively higher, ranging between $600^{\circ} \mathrm{C}$ and $800^{\circ} \mathrm{C}$ under which the lithopone changes its decoloration capability (DC).

At the hot end (head) of the kiln, the left side of the calciner, diesel or petrol is sprayed and burned to generate the heat for the whole calcining and drying process. A thermal sensor is arranged there and the head temperature is normally maintained at about $1200^{\circ} \mathrm{C}$ to assure the heat is enough and stable. As a blower and an exhauster are working at the hot end and cold end, respectively, the air flows from the hot end to cold end, facing up to the material fluid, conveying the heat 
through the kiln, and at the same time taking away the water steam from the material.

Since there is no effective way to detect the output index (DC) directly which has to be measured offline and normally comes out 2 hours later after the lithopone comes out of the exit, the control largely depends on the experienced worker who empirically adjusts the calcination rotary speed according to the calcination temperature. In general, the worker increases the rotary speed if the temperature is high, and vice versa, to ensure the material inside is heated properly.

\section{Data Clustering Algorithm}

Data clustering is the prerequisite for training the ANFIS model and it decides the number of fuzzy rules in the model. There have been different clustering techniques proposed in other literatures [15-18], among which FCM and Subclust are highly regarded and widely adopted.

In FCM, however, the group number has to be given as a premise and iterative process is time consuming. Randomly initialized belongingness matrix leads to uncertainty of the result as well. Also, as for Subclust, since taking data points as candidates, it does not always perform well for finding the optimal centers when the actual centers are not among the data points. For these drawbacks, the author is inspired to find a new clustering technique, aiming at improving not only the accuracy of the result but also the reduced bulk of calculation.

3.1. FCM Algorithm. Consider a set of $n$ data points $\left\{x_{1}, x_{2}\right.$, $\left.\ldots, x_{n}\right\}$ in a $p$-dimensional space, that is, $x_{i}(i=1, \ldots, n)$ is a vector of $p$ coordinates. Given the cluster number $m$, FCM starts by initializing a membership grade $m \times n$ matrix $M$ in random according to (1), indicating the belongingness of each data point to the initial centers.

$$
\begin{aligned}
0 & \leq \mu_{i j} \leq 1, \quad \forall 1 \leq i \leq m, 1 \leq j \leq n, \\
\sum_{i=1}^{m} \mu_{i j} & =1, \quad \forall j=1, \ldots, n,
\end{aligned}
$$

where $\mu_{i j}(1 \leq i \leq m, 1 \leq j \leq n)$ is the degree of membership of $j$ th data point to $i$ th cluster center.

Then new centers are attained and $M$ is upgraded by the following equations, respectively:

$$
x_{k}^{*}=\frac{\sum_{j=1}^{n} \mu_{k j}^{w} x_{j}}{\sum_{j=1}^{n} \mu_{k j}^{w}}, \quad k=1, \ldots, m,
$$

where $x_{k}^{*}$ is the $k$ th cluster center and $w \in[1, \infty)$ is a weighting exponent.

$$
\begin{aligned}
\mu_{i j}=\frac{1}{\sum_{k=1}^{m}\left(\left\|x_{j}-x_{i}^{*}\right\| /\left\|x_{j}-x_{k}^{*}\right\|\right)^{2 /(w-1)}}, & \\
& \quad i=1, \ldots, m ; j=1, \ldots, n,
\end{aligned}
$$

where $\|\cdot\|$ is the Euclidean distance.

This procedure is carried out repeatedly until the cost function $J$ is below a certain tolerance value or no more improvement between the consecutive iterations is noticed. $J$ is defined by

$$
J=\sum_{i=1}^{m} J_{i}=\sum_{i=1}^{m} \sum_{j=1}^{n} \mu_{i j}^{m}\left\|x_{i j}-x_{i}^{*}\right\|^{2}
$$

and $J_{i}$ is cost function for each cluster center, $i=1, \ldots, m$.

3.2. Subclust Algorithm. For the same collection of $n$ data points, Subclust begins with calculating the density value $D$ for each point by the following formula:

$$
D_{i}^{1}=\sum_{i=1}^{n} e^{-\left\|x_{i}-x_{j}\right\|^{2} /\left(r_{a} / 2\right)^{2}}, \quad i=1, \ldots, n,
$$

where $D_{i}^{1}$ is the density value of $i$ th data point at the 1st round of calculation, and $r_{a}$ is a positive constant representing a neighborhood radius. After all the data points are computed, then the point with the highest density value is chosen as the first cluster center $x_{1}^{*}$ and its density value is referred to as $D_{1}^{*}$. Afterwards the calculation goes into the 2 nd round and each point's density value is revised by

$$
D_{i}^{2}=D_{i}^{1}-D_{1}^{*} e^{-\left\|x_{i}-x_{1}^{*}\right\|^{2} /\left(r_{b} / 2\right)^{2}}, \quad i=1, \ldots, n,
$$

where $D_{i}^{2}$ is the density value of $i$ th data point at the 2 nd round of calculation and $r_{b}$ is also a positive constant defining a neighborhood which has measurable reduction in density value. Then the second point with the highest value is attained and if it satisfies some kind of criteria, then it is selected as the 2 nd cluster center. This process repeats until the highest density value is less than a certain threshold. In general, at the $k$ th round of calculation, the equation for computing the density value is

$$
D_{i}^{k}=D_{i}^{k-1}-D_{k-1}^{*} e^{-\left\|x_{i}-x_{k-1}^{*}\right\|^{2} /\left(r_{b} / 2\right)^{2}}, \quad \forall i=1, \ldots, n .
$$

3.3. A New Hybrid Clustering Algorithm Combining FCM and Subclust. A feasible hybrid way is to use the Subclust to obtain the implicit number of clusters and then employ FCM to find their exact centers [19]. But the improvement is rather limited and needs to be further developed. This paper proposes a new way of their combinations which greatly enhance both the computation efficiency and accuracy and it is illustrated in this section.

Considering the above set of data points, first Subclust is adopted to attain $m$ group centers $\left\{x_{1}^{*}, \ldots, x_{m}^{*}\right\}$, and then we use Gaussian function to define a distance grade $m \times n$ matrix $M_{d}$ as follows:

$$
\mu_{i}^{j}=e^{-\left\|x_{j}-x_{i}^{*}\right\|^{2} / 2 \delta^{2}}, \quad i=1, \ldots, m ; j=1, \ldots, n,
$$

where $\mu_{i}^{j}$ represents the relationship between the distance of $j$ th data point and $i$ th cluster center, and $\delta$ is the standard deviation. According to (8), the data point close to a cluster center has a bigger distance grade value. $\delta$ is a key parameter that largely affects the distance grade value. A recommended 
choice is letting $\delta=(0.1 \sim 1) \times r_{a}$. Further ahead, we normalize each column of $M_{d}$ to be the initial membership grade matrix $M_{0}$ :

$$
\mu_{0}^{i j}=\frac{\mu_{i}^{j}}{\sum_{k=1}^{m} \mu_{k}^{j}}, \quad i=1, \ldots, m ; j=1, \ldots, n,
$$

and $\mu_{0}^{i j}$ is initial belongingness of $j$ th data point to $i$ th cluster center.

The next part of the hybrid clustering algorithm is initializing FCM with $M_{0}$. Since $M_{0}$ reflects the actual distance between each point and cluster center, that is, the initial centers are already close to the actual centers, therefore the bulk of computation time in FCM definitely decreases substantially. The holistic procedure of the new clustering algorithm uses the following steps.

Step 1. Normalize the data set $\left\{x_{1}, x_{2}, \ldots, x_{n}\right\}$ in a $p$-dimension space.

Step 2. Find the first cluster center $x_{1}^{*}$ and $D_{1}^{*}$ with (5) being used in the computational process.

Step 3. Revise each point's density value with (6) and find other cluster centers by using the following criteria, supposing $(k-1)$ th $(k \geq 2)$ cluster center has been obtained:

(1) If $D_{k}^{*}<\underline{\varepsilon} D_{1}^{*}$ and $\underline{\varepsilon}$ is a threshold for rejecting a point as a cluster center, go to Step 4 .

(2) If $D_{k}^{*}>\bar{\varepsilon} D_{1}^{*}$ and $\bar{\varepsilon}$ specifies an accepting threshold for a new cluster center, accept $D_{k}^{*}$ as a new cluster center and repeat Step 3.

(3) If $\underline{\varepsilon} D_{1}^{*}<D_{k}^{*}<\bar{\varepsilon} D_{1}^{*}$, accept it as a new cluster center if it satisfies:

$$
\frac{d_{\min }}{r_{a}}+\frac{D_{k}^{*}}{D_{1}^{*}} \geq 1
$$

and $d_{\text {min }}$ represents the shortest distance between $x_{k}^{*}$ and all the previous centers, otherwise reject it and choose the point with the next highest density value and retest according to the above three criteria.

Step 4. Based on the $m$ cluster centers $\left\{x_{1}^{*}, \ldots, x_{m}^{*}\right\}$ found from the previous steps, calculate the distance grade matrix $M_{d}$ with (8) and then the initial membership grade matrix $M_{0}$ with (9).

Step 5. Start FCM by letting $M=M_{0}$.

Step 6. Upgrade the cluster centers using (2).

Step 7. Calculate the cost function according to (4). End the clustering process if $J$ is below a certain tolerance value or the improvement over the previous iteration is less than a certain threshold.

Step 8. Upgrade the belongingness matrix $M$ with (3).

\section{Adaptive Network-Based Inference System (ANFIS)}

ANFIS is produced by Jang [20] and is based on a multilayer feedforward network structure. It has 5 layers with two kinds of nodes: square ones with parameters to be identified and circle ones with none. The directional links between nodes indicate the flow direction of signals.

Consider the system has $p$ inputs $\left\{y_{1}, y_{2}, \ldots, y_{p}\right\}$ and one output $z$ and suppose each input has two fuzzy sets, as seen in Figure 3. The nodes of the same layers have the same function, as described below.

The 1st layer is composed of square nodes with the node function $\mu_{A_{i j}}\left(y_{i}\right)(i=1, \ldots, p ; j=1,2)$, where $y_{i}$ is the input to node $i$ and $A_{i j}$ is a linguistic label representing a fuzzy set. $\mu_{A_{i j}}\left(y_{i}\right)$ is usually chosen among bell-shaped functions and its parameters are referred to as premise parameters.

Every node in the 2nd layer is a circle node with the label $\prod$ which multiplies all the incoming signals from the previous layer and sends the product out:

$$
\omega_{i}=\prod_{A_{i j} \in S_{i}} \mu_{A_{i j}}\left(y_{i}\right)
$$

and $S_{i}$ is the input set of $i$ th node from 1 st layer. $\omega_{i}$ represents the firing strength for $i$ th rule.

The third layer has the same number of circle nodes as the second layer. Each node labeled $N$ calculates the ratio of its input firing strength to the sum of firing strengths in the previous layer:

$$
\bar{\omega}_{i}=\frac{\omega_{i}}{\sum_{j=1}^{m} \omega_{i}} .
$$

Each node of 4 th layer is a square node generating each rule's output:

$$
f_{i}=\sum_{j=1}^{p} a_{i j} y_{j}+b_{i}
$$

and $a_{i j}, b_{i}(i=1, \ldots, m ; j=1, \ldots, p)$ are the set of parameters in this layer and are referred to as consequent parameters.

In the fifth layer, there is only one circle node with the label $\sum$ simply adding all the incoming signals together and producing the overall output $z$ :

$$
z=\sum_{i=1}^{m} \bar{\omega}_{i} f_{i}
$$

The parameters of the network are identified by another hybrid learning procedure, forwards and backwards pass, and the least squares estimate (LSE) formulas and gradient descent method are employed, respectively, in each pass. More details can be found in [20] and applications of ANFIS can be found in $[21,22]$.

\section{Implementation and Results}

Having introduced the hybrid clustering algorithm and ANFIS and their mathematical foundations, this section 


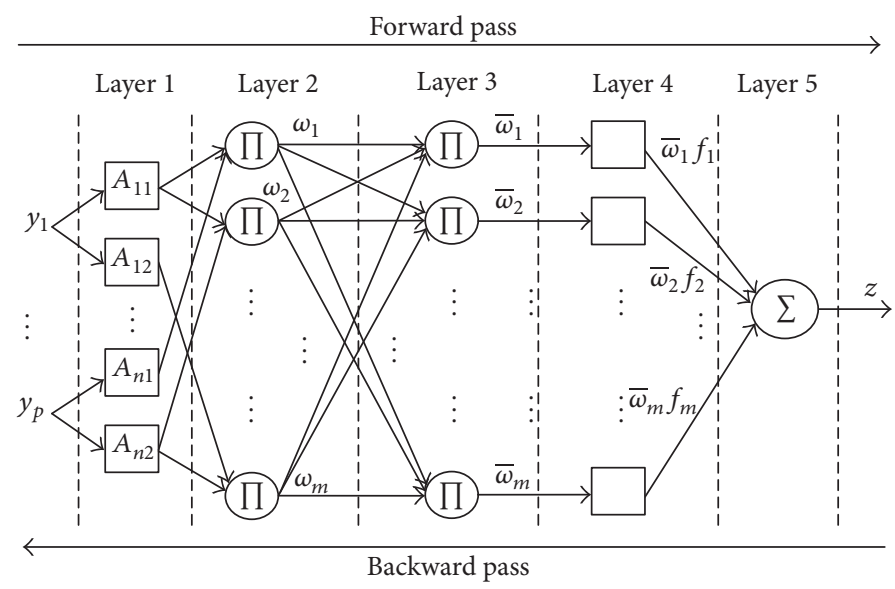

FIgURE 3: ANFIS architecture.

TABLE 1: Value of related parameters of the three clustering algorithms.

\begin{tabular}{lc}
\hline Clustering algorithm & Value of related parameters \\
\hline Subclust & $r_{a}=0.3, r_{b}=1.5 \times r_{a}$ \\
FCM & $w=2$ \\
Hybrid & $r_{a}=0.3, r_{b}=1.5 \times r_{a}, \underline{\varepsilon}=0.15, \bar{\varepsilon}=0.5$, \\
& $\delta=0.5, w=2$ \\
\hline
\end{tabular}

turns back to study the modeling for the calcination process of industrial kiln. First a benchmark group of data is cited to test the three clustering techniques presented in Section 3, and the implementation for modeling is studied afterwards.

5.1. Comparison among Different Clustering Algorithms. A quasi-random two-dimensional data set is used as a benchmark problem to test the performance of the three clustering algorithms. The quasi-random data set is cited from Matlab Toolbox and it includes 140 two-dimension chaotic data points. Assuming there are 3 cluster centers to be found, the three algorithms are implemented individually and their performances are tested. Table 1 lists the value of related parameters in the implementation of the three algorithms.

Figure 4 shows the cluster centers attained by the three methods and it is noticed that the results of FCM and hybrid algorithm are more close to the actual centers. Actually, the root mean square error (RMSE) of Subclust turns out to be
14.7956 which is the highest one. Figure 5 shows the change of cost function over time of FCM and hybrid algorithm and it is evident that the convergence speed of hybrid algorithm prevails over FCM greatly. Table 2 compares the iteration number and RMSE between FCM and hybrid algorithm which also indicates the superior performance of the hybrid algorithm to FCM.

5.2. Calcination Process Modeling. The first question to be solved is the determination of the input and output variables for the control model. The method undertaken in this paper is to rely on the experience of the sophisticated workers and the analysis on the calcination mechanism inside the kiln. In practice, the worker regulates the calcination rotary speed $R$ $(\mathrm{Hz})$ according to the calcination temperature $T\left({ }^{\circ} \mathrm{C}\right)$, as seen in Figure 1, which provides important information that $R$ can be the only output and $T$ should be one of the input variables.

A further study at the inside calcination process manifests that the material changes its property to meet the quality requirement, that is, DC, mainly when it is going through the inner pot because the temperature there is much higher than other parts inside the kiln. This process normally takes 15 to 20 minutes depending on the rotary speed $R$. Consequently, the calcination temperature $T$ and rotary speed $R$ in the previous time phase should also be considered into the input variables of the model, which matches the time-delay property of the calcination process. After testing different combinations of $T$ and $R$ in their previous time phases, a set of inputs is chosen as below:

$$
Y=[T(k) T(k-1) T(k-2) T(k-3) R(k-1) R(k-2) R(k-3)]
$$

where $k$ is the time index and the time interval between the two successive time indexes is 5 minutes.

The model for the calcination process is then built up by adopting ANFIS coupled with the novel hybrid clustering algorithm proposed in Section 3. A group of 600 consecutive data points, coming from successful control of sophisticated workers, are chosen to train and test the model, among which 400 are used as training data and 200 are used as checking 


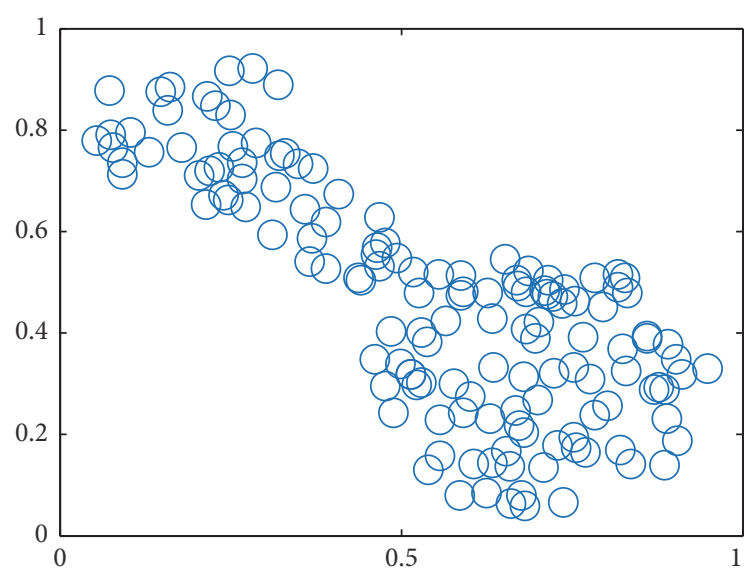

(a)

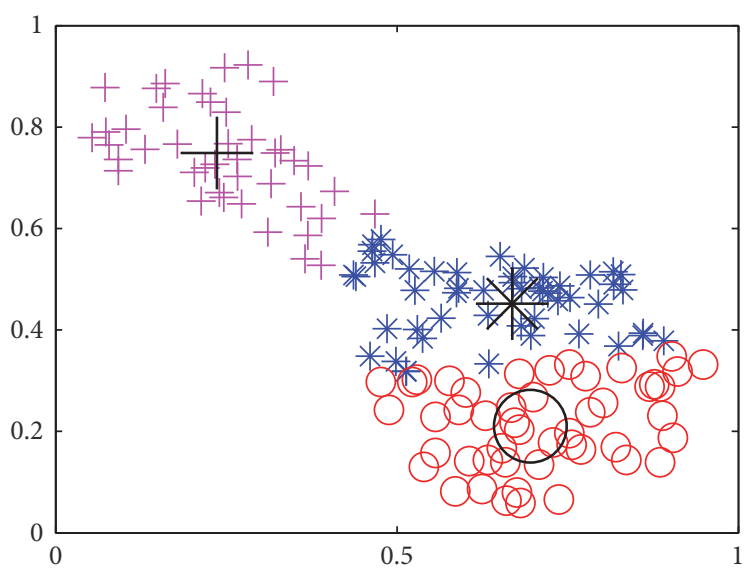

(c)

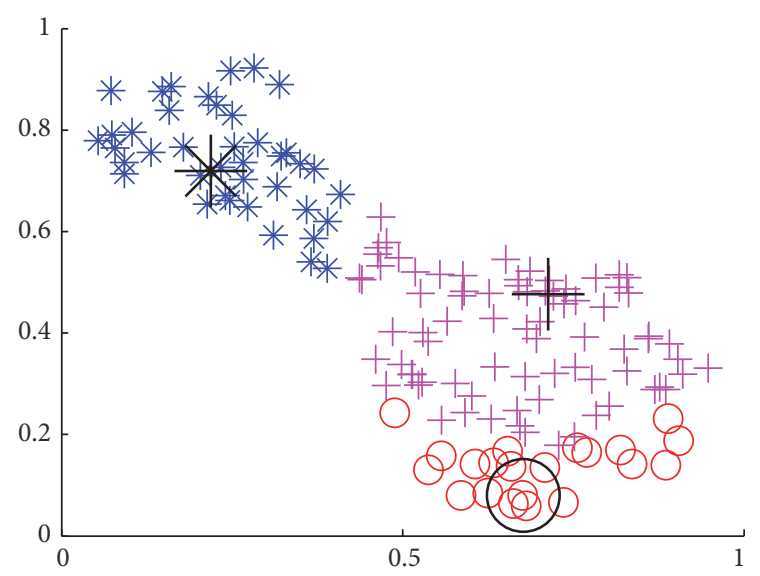

(b)

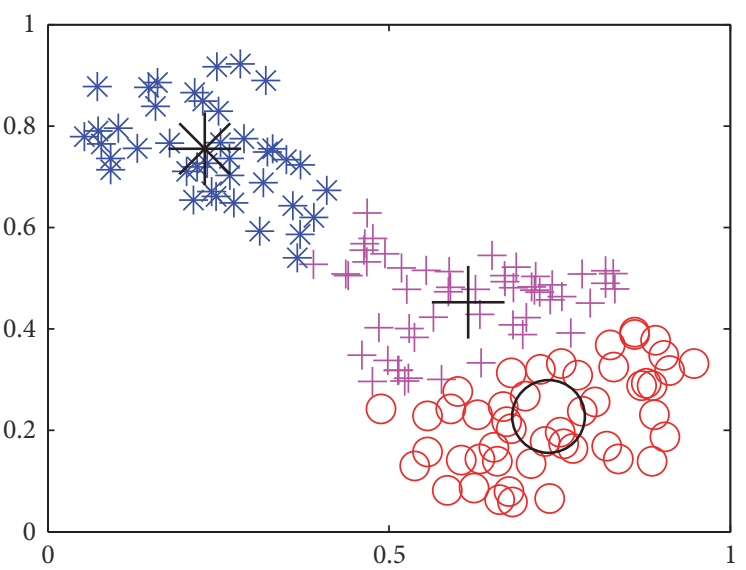

(d)

Figure 4: Cluster centers from different algorithms on the quasi-random data. (a) The quasi-random data. (b) Cluster centers from Subclust. (c) Cluster centers from FCM. (d) Cluster centers from hybrid algorithm.

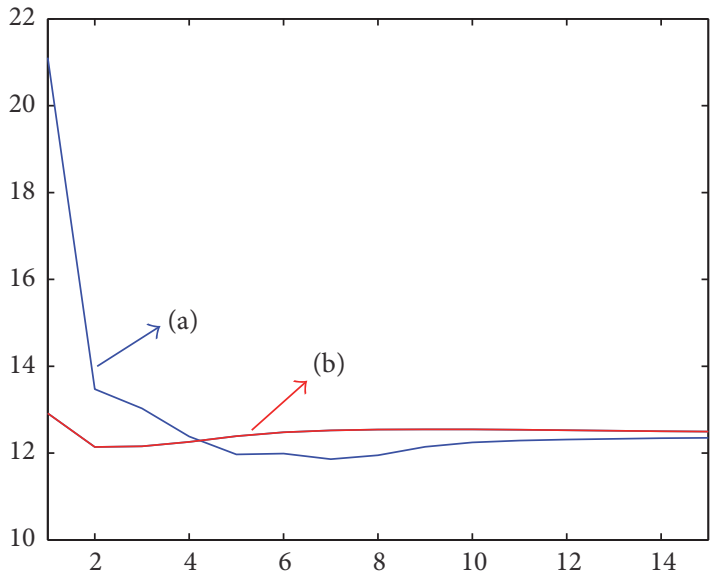

(a) FCM

— (b) Hybrid

FIGURE 5: Plots of cost function of FCM and hybrid algorithm.

data. In the clustering stage, related parameters are selected as $r_{a}=0.3081, r_{b}=1.25 \times r_{a}, \underline{\varepsilon}=0.15, \bar{\varepsilon}=0.5, \delta=0.5$,
TABLE 2: Clustering performance of FCM and hybrid algorithm.

\begin{tabular}{lcc}
\hline Performance & FCM & Hybrid Subclust \\
\hline Iteration times & 7 & 2 \\
RMSE & 14.3025 & 13.7007 \\
\hline
\end{tabular}

and $w=2$, which yields 11 rules for ANFIS. Figure 6 shows the outputs of the model after a training of 250 epochs and it is noticed that the model works satisfactorily on both the training data and checking data.

A further comparison is made between ANFIS with FCM clustering and ANFIS with the new hybrid algorithm. A longer training as 300 epochs is applied for ANFIS this time. During the process, the cost function on clustering phase and checking data error for ANFIS are checked, respectively, as seen in Figures 7 and 8. It can be seen that both the cost function and checking data error in the method of ANFIS with the new hybrid algorithm are smaller at each epoch and converge more quickly. Detailed performance for these two methods is listed in Table 3. 


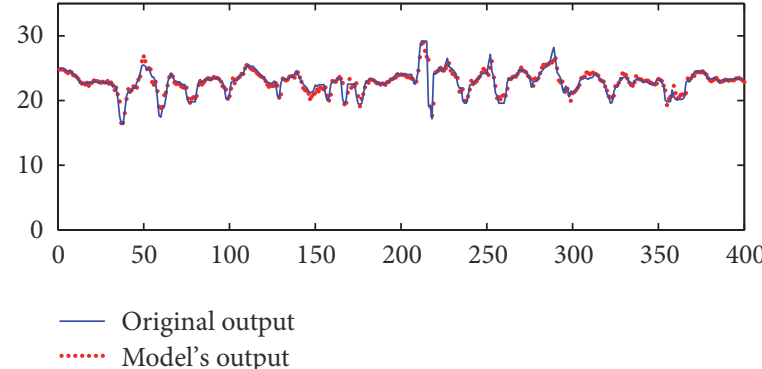

(a)

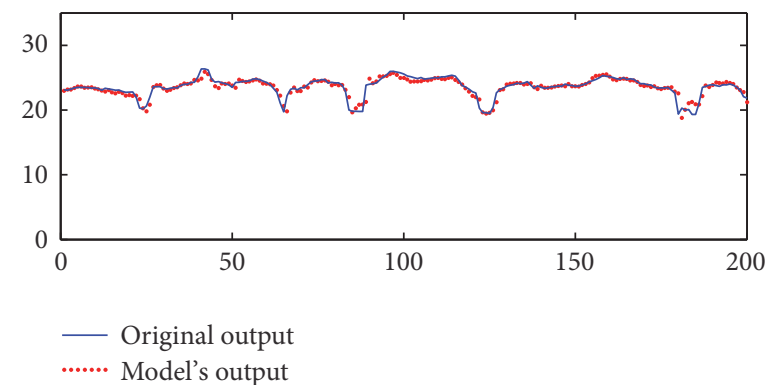

(b)

FIgURE 6: Plots of the model's output. (a) Output on training data. (b) Output on checking data.

TABle 3: Performance comparison between ANFIS with FCM and ANFIS with the new hybrid method.

\begin{tabular}{lcc}
\hline Performance & ANFIS with FCM algorithm & ANFIS with the new hybrid algorithm \\
\hline Iterations of clustering & 25 & 5 \\
Mean of training & 0.3923 & 0.3262 \\
Mean of checking & 0.4214 & 0.3943 \\
RMSE of training & 0.0321 & 0.0279 \\
RMSE of checking & 0.0467 & 0.0459 \\
\hline
\end{tabular}

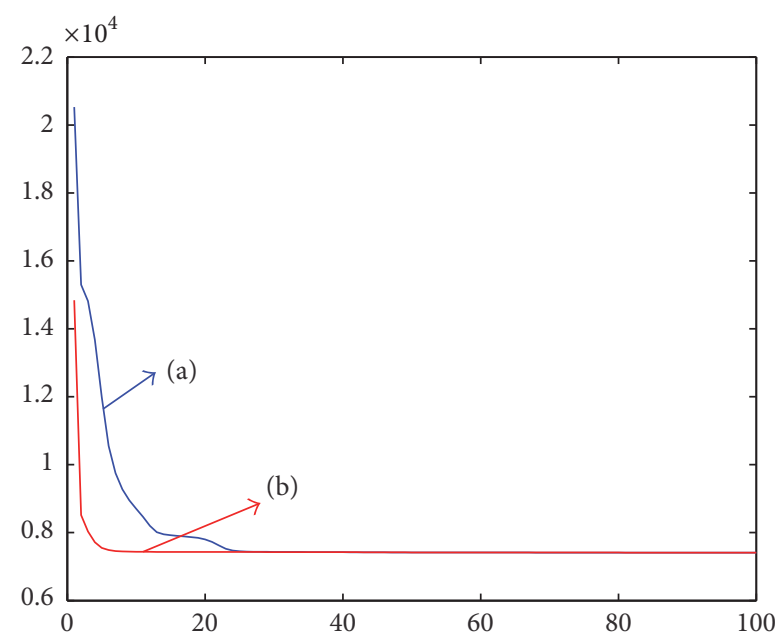

(a) FCM

(b) Hybrid

FIGURE 7: Plots of cost function on clustering phase.

\section{Conclusion}

A novel hybrid clustering algorithm combining FCM with Subtractive Clustering Method is proposed and is proved to be more efficient with reduced computation and it leads to more accuracy for the clustering result. ANFIS is employed to establish the control model for the calcination process of industrial rotary kiln with a satisfactory outcome and it sets a role model for similar control situations in industrial field. Coupled with the new hybrid clustering algorithm, the performance of ANFIS improves greatly with reduced computation on clustering phase and approaches more accuracy

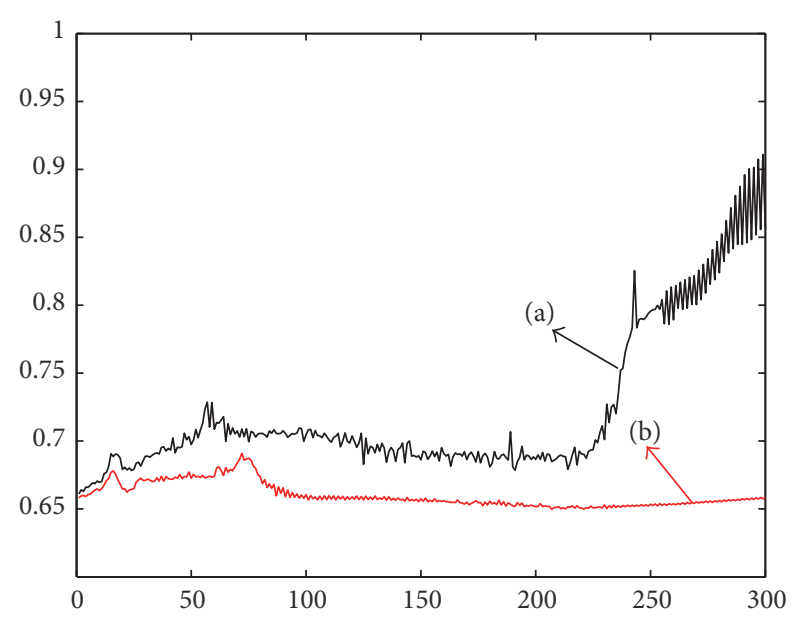

(a) ANFIS with FCM

(b) ANFIS with hybrid

FIGURE 8: Error plots on checking data for ANFIS.

to the original outputs. Furthermore, study can be focused on the issue of determining the number of time phases and the time interval in the input vector $Y$, since it is mainly decided empirically currently. Also, the effect from the drying part of the rotary kiln on modeling is neglected in this paper; the roles of drying temperature and drying rotary speed on the model are to be into consideration as well.

\section{Competing Interests}

The author declares that there is no conflict of interests regarding the publication of this paper. 


\section{Acknowledgments}

This research is partially supported by National Natural Science Foundation of China (61403264), partially supported by Program of Cultivation for Outstanding Young Scholars and "Thousand, Hundred and Ten" Teacher Cultivation Program of Guangdong Province (ZX03240302), and partially supported by Program of Building Brand Major of High Vocational Education of Guangdong Province.

\section{References}

[1] M. Järvensivu, E. Juuso, and O. Ahava, "Intelligent control of a rotary kiln fired with producer gas generated from biomass," Engineering Applications of Artificial Intelligence, vol. 14, no. 5, pp. 629-653, 2001.

[2] M. Järvensivu, K. Saari, and S.-L. Jämsä-Jounela, "Intelligent control system of an industrial lime kiln process," Control Engineering Practice, vol. 9, no. 6, pp. 589-606, 2001.

[3] N. Q. Dinh and N. V. Afzulpurkar, "Neuro-fuzzy MIMO nonlinear control for ceramic roller kiln," Simulation Modelling Practice and Theory, vol. 15, no. 10, pp. 1239-1258, 2007.

[4] M. Georgallis, P. Nowak, M. Salcudean, and I. S. Gartshore, "Modelling the rotary lime kiln," The Canadian Journal of Chemical Engineering, vol. 83, no. 2, pp. 212-223, 2005.

[5] Z. Söğüt, Z. Oktay, and H. Karakoç, "Mathematical modeling of heat recovery from a rotary kiln," Applied Thermal Engineering, vol. 30, no. 8-9, pp. 817-825, 2010.

[6] Y. H. Kim, "Development of process model of a rotary kiln for volatile organic compound recovery from coconut shell," Korean Journal of Chemical Engineering, vol. 29, no. 12, pp. 16741679, 2012.

[7] Y. Wang, X.-H. Fan, and X.-L. Chen, "Mathematical models and expert system for grate-kiln process of iron ore oxide pellet production (Part I): mathematical models of grate process," Journal of Central South University of Technology, vol. 19, no. 4, pp. 1092-1097, 2012.

[8] X.-H. Fan, Y. Wang, and X.-L. Chen, "Mathematical models and expert system for grate-kiln process of iron ore oxide pellet production. Part II: rotary kiln process control," Journal of Central South University of Technology, vol. 19, no. 6, pp. 17241727, 2012.

[9] Y. Cai, "Research on soft measurement modeling for industry rotary kiln based on flexible neural network," in Proceedings of the International Conference on Computer Science and Electronics Engineering (ICCSEE '12), pp. 343-346, IEEE, Hangzhou, China, March 2012.

[10] T. Zhongda, L. Shujiang, W. Yanhong, and W. Xiangdong, "A multi-model fusion soft sensor modelling method and its application in rotary kiln calcination zone temperature prediction," Transactions of the Institute of Measurement and Control, vol. 38, no. 1, pp. 110-124, 2016.

[11] L. Zhang, C. Zhang, Q. Xu, and C. Wang, "Modelling of lime kiln using subspace method with new order selection criterion," Mathematical Problems in Engineering, vol. 2014, Article ID 816831, 11 pages, 2014.

[12] M. Badoni, A. Singh, and B. Singh, "Adaptive neurofuzzy inference system least-mean-square-based control algorithm for DSTATCOM," IEEE Transactions on Industrial Informatics, vol. 12, no. 2, pp. 483-492, 2016.
[13] P. García, C. A. García, L. M. Fernández, F. Llorens, and F. Jurado, "ANFIS-Based control of a grid-connected hybrid system integrating renewable energies, hydrogen and batteries," IEEE Transactions on Industrial Informatics, vol. 10, no. 2, pp. 1107-1117, 2014.

[14] K. S. Ajil, P. K. Thapliyal, M. V. Shukla, P. K. Pal, P. C. Joshi, and R. R. Navalgund, "A new technique for temperature and humidity profile retrieval from infrared-sounder observations using the adaptive neuro-fuzzy inference system," IEEE Transactions on Geoscience and Remote Sensing, vol. 48, no. 4, pp. 1650-1659, 2010.

[15] J. C. Bezdek, R. Ehrlich, and W. Full, "FCM: the fuzzy c-means clustering algorithm," Computers and Geosciences, vol. 10, no. 23, pp. 191-203, 1984.

[16] S. L. Chiu, "Fuzzy model identification based on cluster estimation," Journal of Intelligent and Fuzzy Systems, vol. 2, no. 3, pp. 267-278, 1994.

[17] Q. Du, Analysis and research on process control for a lithopone calcination rotary kiln [Ph.D. thesis], South China University of Technology, 2008.

[18] A. K. Jain and R. C. Dubes, Algorithms for clustering data, Prentice Hall Advanced Reference Series, Prentice Hall, Inc., Englewood Cliffs, NJ, 1988.

[19] Q. Yang, D. Zhang, and F. Tian, "An initialization method for fuzzy c-means algorithm using subtractive clustering," in Proceedings of the 3rd International Conference on Intelligent Networks and Intelligent Systems (ICINIS '10), pp. 393-396, Shenyang, China, November 2010.

[20] J.-S. R. Jang, "ANFIS: adaptive-network-based fuzzy inference system," IEEE Transactions on Systems, Man and Cybernetics, vol. 23, no. 3, pp. 665-685, 1993.

[21] K. Tan, Y. Ye, Q. Cao, P. Du, and J. Dong, "Estimation of arsenic contamination in reclaimed agricultural soils using reflectance spectroscopy and ANFIS model," IEEE Journal of Selected Topics in Applied Earth Observations and Remote Sensing, vol. 7, no. 6, pp. 2540-2546, 2014.

[22] S. A. Khan, M. D. Equbal, and T. Islam, "A comprehensive comparative study of DGA based transformer fault diagnosis using fuzzy logic and ANFIS models," IEEE Transactions on Dielectrics and Electrical Insulation, vol. 22, no. 1, pp. 590-596, 2015. 


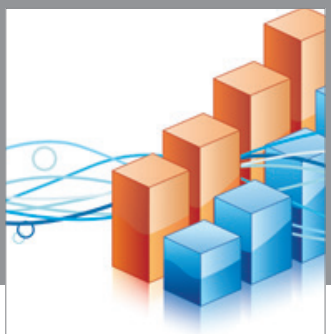

Advances in

Operations Research

vatem alat4

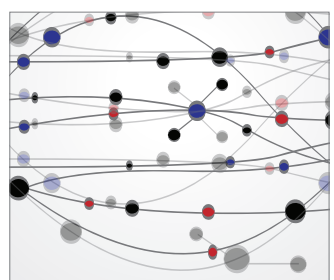

\section{The Scientific} World Journal
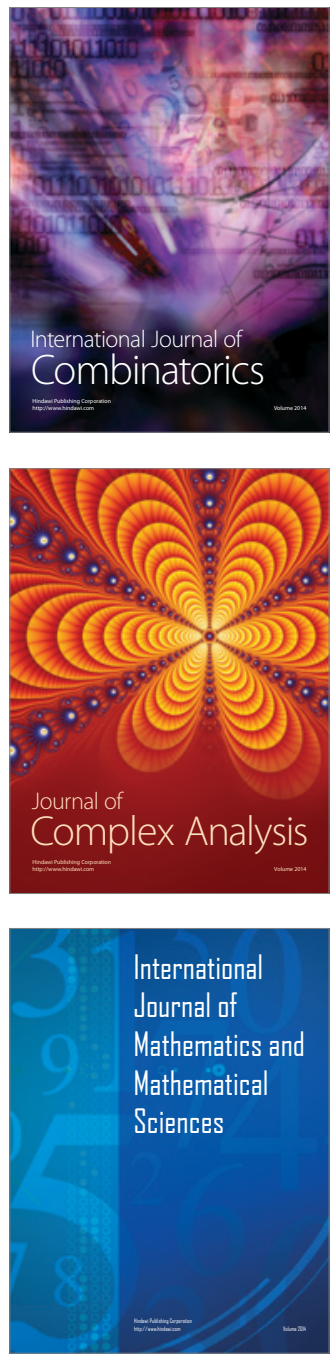
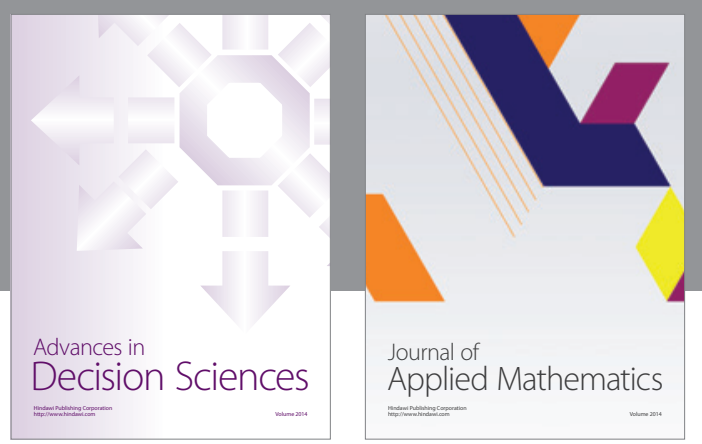

Algebra

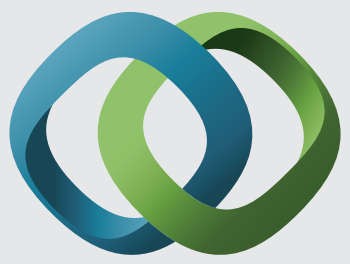

\section{Hindawi}

Submit your manuscripts at

https://www.hindawi.com
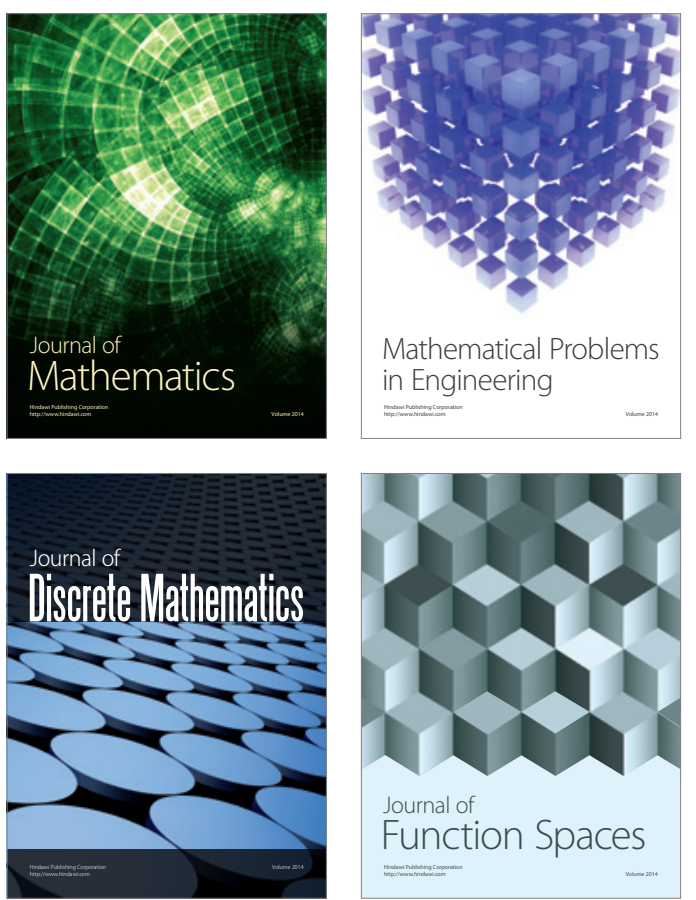

Mathematical Problems in Engineering
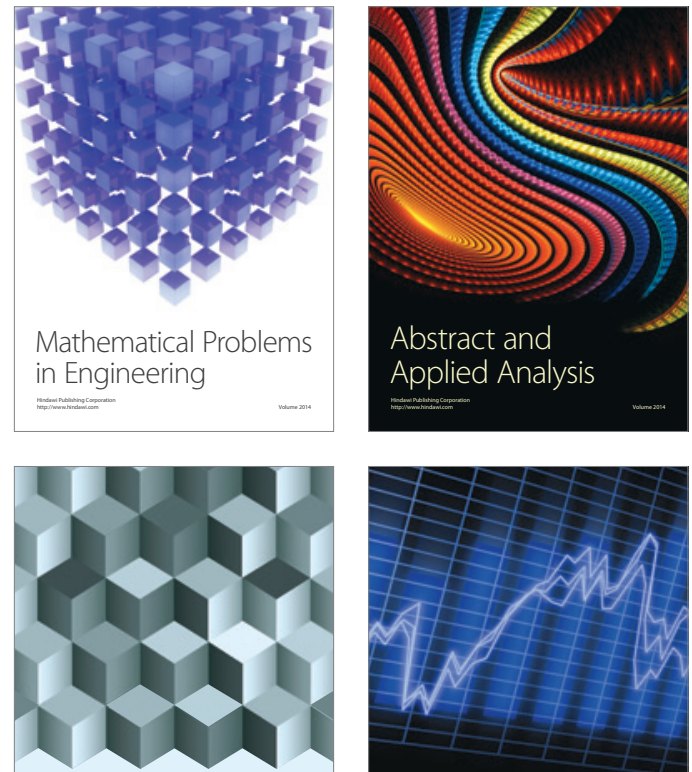

Journal of

Function Spaces

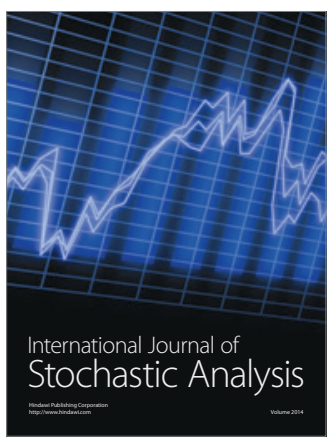

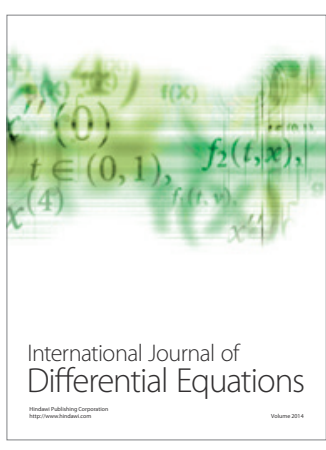
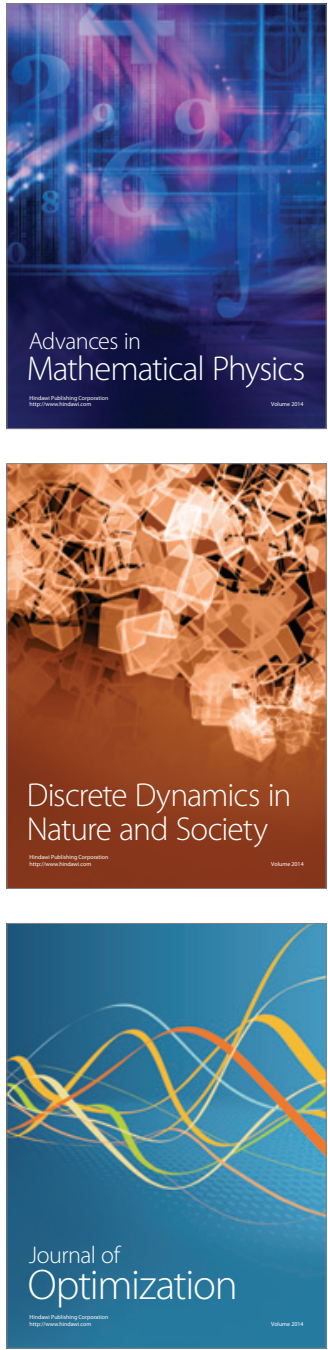